\title{
RESEARCH ON PERFORMANCE TEST AND EVALUATION METHOD OF ASSEMBLY PACKAGING AMMUNITION
}

\author{
Wenzhao Li, Wei Wang, Min Gao, Junbo Wang \\ Ordnance Engineering College, Shijiazhuang, China; Email.sharpshaw@sina.com
}

\begin{abstract}
In order to evaluate accurately the comprehensive performance of assembly packaging ammunition using small samples, a new test and evaluate method is presented. Firstly carry out the consistency test on the performance parameters of electronic parts of ammunition before and after the experiment, which are liable to the influence of various environmental factors, if the result of test is consistent, then firing test is carried out in order to check the reliability of whole ammunition. In the course of the consistency test, according to the difference of electric parameters rank sum test and T-test is used, and the Bayes method is applied in the whole ammunition's reliability evaluation, and an example is demonstrated in order to verify the validity of this test and evaluation method
\end{abstract}

Key words: Ammunition packaging, Small samples, Rank sum test, T-test, Bayes method.

\section{INTRODUCTION}

For testing the quality of inner ammunition in general, all kinds of environmental test on assembly packaging ammunition should be carried out according to the demand of ammunition support. By virtue of the difference of experiment's method, packaging test can be divided into two kinds, one is static test, such as stack test, pressure test, temperature test, humidity test etc, the other is dynamic test, such as falling test, impacting test, collision test and others. After doing traditional tests, firing test must be put up to analysis the protecting ability of the packaging material and the rationality of structure. But the test samples of new armed ammunition which is expensive

Please use the following format when citing this chapter:

Li, Wenzhao, Wang, Wei, Gao, Min, Wang, Junbo, 2006, in International Federation for Information Processing (IFIP), Volume 207, Knowledge Enterprise: Intelligent Strategies In Product Design, Manufacturing, and Management, eds. K. Wang, Kovacs G., Wozny M., Fang M., (Boston: Springer), pp. 818-824. 
is small, it is very difficult to get exactly the performance of packaging with common methods ${ }^{[1,2]}$.

From the result of research on the design and production process, in expensive ammunition we can see the quality of inner electronic parts are liable to the influence of various environmental factors compared with others in the same condition. The states of most electronic parts can be checked through special test window, therefore we can get the data of performance before and after traditional packaging test experiment easily. If the difference between two results is distinct, we can draw a conclusion that packaging is not reasonable, if the results are consistent, then firing test is carried out to test the reliability of whole ammunition in order to judge whether it reaches an appointed technical index.

\section{EXPERIMENT SCHEME}

At first we select $n$ ammunition samples from a batch of accepted products at random and test electrical parameters, and get the test results, which is denoted as: $X=\left(X_{1}, X_{2}, \cdots, X_{n}\right)$. Then the environmental stress test is carried out which includes stack test, pressure test, temperature test, humidity test, falling test, impacting test, collision test etc. At last we take out the ammunition tested with stress experiment from package box, and test each one's electrical parameter again, which is denoted as $Y=\left(Y_{1}, Y_{2}, \cdots, Y_{n}\right)$.

Analyze the consistency of $X$ and $Y$ with the statistical method, if the difference of the two results is distinct, the quality of the packaging is not reasonable, otherwise if the result is compatible, then the firing test is carried out in order to verify the quality of the whole ammunition further. Through the firing test, the result function $(s, f)$ is obtained, the variable $s$ indicates the number of success, $f$ indicates the number of failure. Given the degree of confidence $\gamma$, we can figure out the lower level of the reliability $R_{L}$ with the formula $(s, f)$, and compare $R_{L}$ with the ammunition's reliability technical index $R_{L}^{\prime}$. By above way, we can judge the level of the ammunition's reliability is remarkable or not, and the conclusion of the packaging test is got. The experiment scheme is shown in figure 1. 


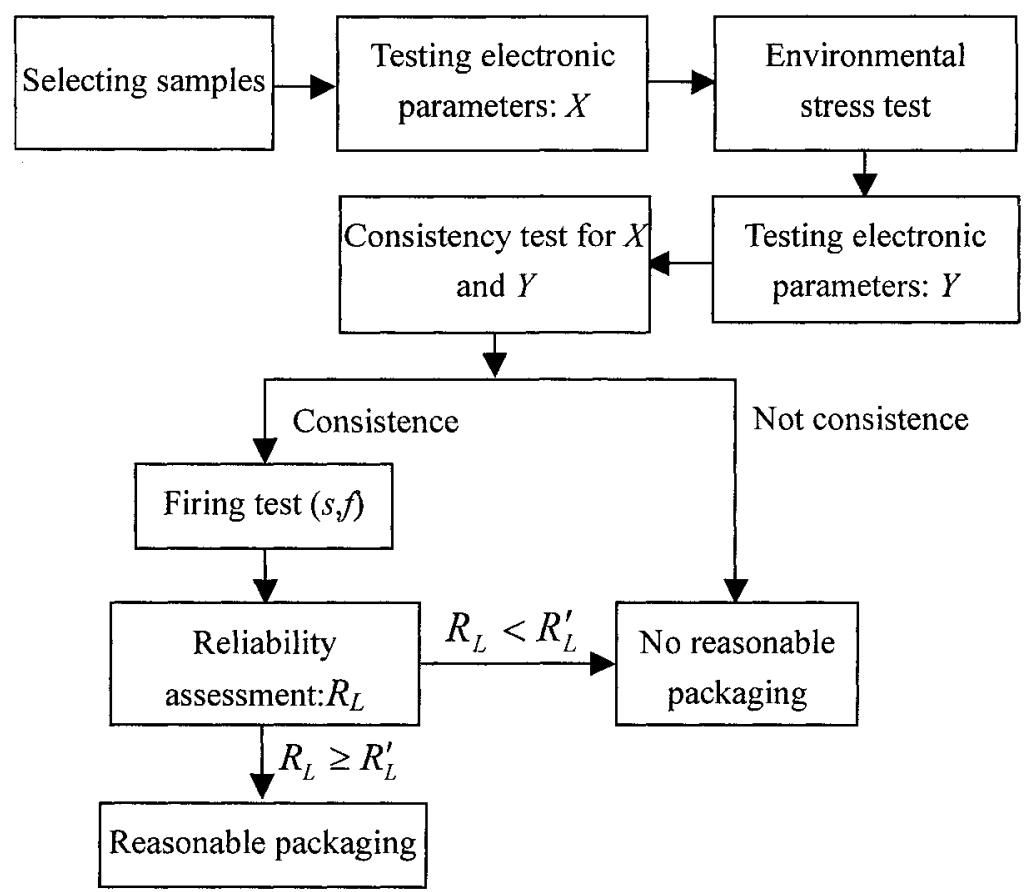

Figure I Experiment scheme

3. STATISTIC ANALYSIS METHOD OF DATA

\subsection{Test method of consistency}

\subsubsection{T-test of two collectivities}

Given $X_{1}, X_{2}, \cdots, X_{n}$ and $Y_{1}, Y_{2}, \cdots, Y_{m}$ are two different samples selected from continuous collectivity $F(x) \sim N\left(\mu_{1}, \sigma^{2}\right)$ and $G(x) \sim N\left(\mu_{2}, \sigma^{2}\right)$. For checking the consistency of $X$ and $Y$, that is, the hypothesis is as follows: $H_{0}: \mu_{1}=\mu_{2} ; H_{1}: \mu_{1} \neq \mu_{2}$.

we sign: 


$$
S=\left\{(n+m) \frac{\sum_{i=1}^{n}\left(X_{i}-\bar{X}\right)^{2}+\sum_{i=1}^{m}\left(Y_{i}-\bar{Y}\right)^{2}}{(n+m-2) n m}\right\}^{1 / 2}
$$

Given a check level $\alpha$, the formula (2) is set up.

$$
\left\{\begin{array}{l}
|\bar{X}-\bar{Y}|>S t_{n+m-2}(\alpha / 2) \Rightarrow \text { Reject } \\
|\bar{X}-\bar{Y}|<S t_{n+m-2}(\alpha / 2) \Rightarrow \text { Accept. }
\end{array}\right.
$$

\subsubsection{Rank sum test}

Given $X_{1}, X_{2}, \cdots, X_{n}$ and $Y_{1}, Y_{2}, \cdots, Y_{m}$ are two different samples selected from continuous collectivity $F(x)$ and $G(x)$. For checking the consistency of $X$ and $Y$, that is, the hypothesis is as follows: $H_{0}: F(x)=G(x)$.

Put $X$ and $Y$ together, and arrange these data in order from small to big. The serial number of each data is called rank. Sum the rank of small samples compared with $X$ and $Y$, denoted as $T$. If the hypothesis $H_{0}$ is true, the difference of two collectivities $F(x)$ and $G(x)$ is no distinct, and the value of $T$ will be suitable.

Lookup the rank sum table of Wilcoxon ${ }^{[3,4]}$, we can get the critical value $T_{1}$ and $T_{2}$ under the test level $\alpha$.

$$
P\left\{T_{1}<T<T_{2}\right\}=1-\alpha
$$

If $T_{1}<T<T_{2}$, we say the hypothesis $H_{0}$ is true, and the difference between $F(x)$ and $G(x)$ is not distinct. If $T \leq T_{1}$ or $T \geq T_{2}$, we say the hypothesis $H_{0}$ is false, and the difference between $F(x)$ and $G(x)$ is distinct. If the value of $n$ and $m$ is larger than 10 , the statistical variable $T$ belongs to the normal distribution, this is:

$$
T \sim N\left(\frac{n(n+m+1)}{2}, \frac{n m(n+m+1)}{12}\right)
$$


Therefore we can use $U$ check method, and select statistical variable is shown bellow.

$$
U=\frac{T-\frac{n(n+m+1)}{2}}{\sqrt{\frac{n m(n+m+1)}{12}}} \sim N(0,1)
$$

Under the check level $\alpha$, the rejection region $W$ is as follows.

$$
W=\left\{|u|>u_{1-\alpha / 2}\right\}
$$

We can use software such as SAS, GAUSS, SPSS, MATLAB etc to help us calculate the number of $T$ easily. In this paper, we use the rank sum function $[\mathrm{p}, \mathrm{h}]=\operatorname{ranksum}(\mathrm{x}, \mathrm{y}, \mathrm{alpha})$ in MATLAB to do this work, in which the variable $P$ is shown below.

$$
p=P\left\{T_{1}<T<T_{2}\right\}
$$

If the value of $P$ is larger than the given level, we reject $H_{0}$ and draw a conclusion that the difference of two collectivities is distinct, otherwise we accept $H_{0}$ and draw a consistent conclusion.

\subsection{Method of reliability assessment}

The result of the reliability assessment is explained with the lower limit of the reliability $R_{L}$, that is, given the degree of confidence $r$ the equation $P\left\{R \geq R_{L}\right\}=r$ is true, in which the variable $R$ is the reliability of system.

If the collectivity belong to the distribution $B(1, R)$, the test result is false or true only, we select $n$ products as test samples at random, and the number of false result $X$ meets the equation (8).

$$
P\{X=k\}=C_{n}^{k} R^{n-k}(1-R)^{k} \quad k=0,1, \cdots, n
$$

If we select Bayes method to assess the reliability of system, the distribution $\operatorname{Beta}(a, b)$ is adopted, in which it's density function is shown as follows.

$$
\pi(R)=\frac{\Gamma(a+b)}{\Gamma(a) \Gamma(b)} R^{a-1}(1-R)^{b-1} \quad 0 \leq R \leq 1
$$


In above function the variable $a$ and $b$ is the super parameters of distribution before testing. After we get $\operatorname{Bet} a(a, b)$ and the test samples $(s, f)$, by the Bayes theory calculate the distribution after test $\pi(R \mid s, f)=\operatorname{Beta}(a+s, b+f)$. So the lower limit $R_{L B}$ under the level of $\gamma$ can be gotten by the formula (10).

$$
\int_{0}^{R_{L B}} \pi(R \mid s, f) d R=I\left(R_{L B} ; a+s, b+f\right)=1-\gamma
$$

Parameters $a$ and $b$ is confirmed with the information before test. Usually for the ammunition product, $(a, b)$ is set $(1 / 2,1 / 2)^{[5,6]}$.

\section{APPLICATION EXAMPLE}

In order to test the packaging quality of one type missile, according to the test and accepting rules of ammunition, select eight products as test samples in qualified products. By special measurement instrument, 28 different photoelectricity parameters of each product were attained, in which 16 parameters belong to the distribution of $B(1, R)$ and they need not test. Among all parameters only 12 connect with the quality of ammunition closely, in which there are 8 parameters belong to the normal distribution and we use T-test to check their consistency before and after experiment, use rank sun test to check other 4 parameters which the distribution is unknown. If one of 12 items checked parameters is distinct, it is shown the quality of packaging will not be qualified. Table 1 is the test data of winding's voltage of $\mathrm{OK}_{1}$, which belong to the normal distribution and Table 2 is the test data of EBT resistance values. The analysis procedure is shown as follows.

Table I The Voltage of $\mathrm{OK}_{1}(\mathrm{~V})$

\begin{tabular}{ccccccccc}
\hline Results before test $X$ & 0.11 & -0.09 & 0.16 & 0.27 & 0.20 & 0.08 & 0.22 & 0.03 \\
Results after test $Y$ & -0.10 & 0.10 & 0.28 & -0.16 & 0.08 & 0.19 & -0.20 & -0.39 \\
\hline
\end{tabular}

Under the check level of $\alpha=0.05$, we get $\bar{X}-\bar{Y}=0.1475$ and $S=0.96981$ with the formula (1). Lookup the T-test table, we get $t_{14}(0.975)=2.14479$, because of $|\bar{X}-\bar{Y}|<S t_{14}(0.975)$, the difference before and after test is not distinct. 
Table 2 The resistance values of EBT $(\Omega)$

\begin{tabular}{lllllllll}
\hline Results before test $X$ & 3.5 & 3.0 & 3.3 & 2.7 & 1.5 & 4.3 & 2.2 & 1.3 \\
Results after test $Y$ & 2.9 & 4.5 & 2.0 & 3.6 & 1.9 & 1.8 & 1.6 & 2.6 \\
\hline
\end{tabular}

Under the check level of $\alpha=0.05$, we get the value of variable $T$ is 70 . Lookup the Rank sum test table, we get $T_{1}=52$ and $T_{2}=84$. Because $T_{1}<T<T_{2}$, the difference before and after test is not distinct.

According to above method, under the level of $\alpha=0.05,12$ test parameters are all not distinct. Then the firing test is carried out, and the result is: the success number $s$ is 8 and the failure number $f$ is 0 , given the degree of confidence $\gamma$ is 0.8 , we calculate $R_{L}$ with the formula (10), $R_{L}=0.90532$. Compare with the designed index $R_{L}^{\prime}=0.9(\gamma=0.8)$, we can get the conclusion that the quality of ammunition is good and the protecting ability of ammunition packaging is eligible.

\section{CONCLUSIONS}

Because of the particularity of ammunition, it is not easy thing very much for assessing and testing the integral performance of the assembly packaging ammunition, so this paper presents a problem of testing the performance of the assembly packaging ammunition under the condition of small samples. About this problem, a method of connecting checking and assessment is introduced, and an example is demonstrated in order to verify the validity of this method, which can evaluate the integral performance of ammunition packaging accurately and offer a new mathematics theory method and path for testing the integral performance of ammunition.

\section{REFERENCES}

1. Jinhuai Zhang. Ordnance Equipment Small Sample Test Analysis and Assessment. Defence industrial press, 2001

2. Efron.B.. Bootstrap Methods: Another Look at Jackknife. The Annals of Statistical, 1979, 7 : $1-26$

3. Xiru Chen. Introduction of Mathematic Statistics. Science and technology press, 1997

4. Fengqin Xu. A Summary of Chain Sampling Plans(Part I). Mathematic Statistics and Management, 1999, 18(2): 34-39

5. Fengqin Xu. A Summary of Chain Sampling Plans(Part II). Mathematic Statistics and Management, 1999, 18(4): 38-42

6. Shisong Mao. Bayes Mathematic Statistics. China Statistic Press, 1999. 